\title{
ANALYTICAL MODEL OF THE ANISOTROPIC DIMENSIONAL CHANGE ON SINTERING OF FERROUS PM PARTS
}

\author{
I. Cristofolini, N. Corsentino, M. Larsson, A. Molinari
}

\begin{abstract}
This work proposes an analytical model developed from experimental data to describe the anisotropic dimensional change on sintering. Axialsymmetric iron parts differing for geometry and sintering conditions have been investigated, aiming at highlighting the influence of geometry. The specimens were measured in the green and sintered state by a coordinate measuring machine (CMM). The dimensional changes of height, external diameter and internal diameter were derived from measurement results. The anisotropy of the dimensional variations has been studied with reference to the isotropic dimensional change derived from the change in volume of the parts. The influence of geometry and sintering temperature was highlighted. To properly describe the dimensional variations in the compaction plane, the dimensional change of the external diameter versus the dimensional change of the internal one has been analysed. By means of the experimental data, a reliable analytical relationship has been found, dependent on the parts geometry. An anisotropy parameter has been identified, which allows relating the dimensional change in the compaction plane and in the axial direction to the isotropic dimensional change. This parameter depends both on geometry and on sintering conditions. By means of the anisotropy parameter an analytical model for the anisotropic behaviour has been developed.
\end{abstract}

Kewords: shrinkage anisotropy, geometry

\section{INTRODUCTION}

Anisotropic dimensional change on sintering is a well-known phenomenon in the PM industry. In the conventional press and sinter process, the dimensional changes on sintering along the directions parallel and perpendicular to the direction of compaction may significantly differ. Morphological and microstructural modifications, in fact, may be induced in the green part by the difference between axial and radial pressure in die pressing [1]. These modifications, and the inhomogeneous packing of the solid particles, are mainly responsible for the anisotropic dimensional change in solid state sintering, while in liquid phase sintering a significant influence of the uneven distribution of the liquid phase is observed. In Fe-Cu alloys the larger swelling was observed in the axial direction [2], while in $\mathrm{Fe}-\mathrm{Cu}-\mathrm{C}$ alloys the direction of prevailing swelling depends on $\mathrm{Cu}$ content $[3,4]$. The prevailing effect of early stages of sintering in the solid state on anisotropy and its attenuation due to the formation of the liquid phase were also observed by dilatometric analysis in $\mathrm{Fe}-\mathrm{P}-\mathrm{Cu}$ [5] and $\mathrm{Fe}-\mathrm{Cu}-\mathrm{C}$ alloys [6]. The contribution of gravity to the anisotropy of dimensional variations under both solid and liquid phase sintering has been

Ilaria Cristofolini, Nicolò Corsentino, Alberto Molinari. University of Trento - Department of Industrial Engineering - 38122 Trento, Italy

Mats Larsson, Höganäs AB - SE-263 83 Höganäs, Sweden 
discussed, also proposing both an analytical and a numerical finite element analysis [7,8]. An additional effect of friction with sintering trays was also observed [9]. In previous works the influence of anisotropic dimensional changes on the precision of sintered parts has been studied. Both swelling [6, 10] and shrinking systems $[\underline{5}, 11]$ have been investigated, also evaluating the attainable dimensional tolerances. The mechanism responsible for the anisotropic dimensional change during sintering has been investigated by dilatometry, also highlighting the relationship with the deformation of particles due to prior compaction [12]. The effect of geometry on the anisotropic dimensional changes has been investigated on different materials and a preliminary model to describe the anisotropic behaviour has been developed $[13,14]$. On the basis of this model, a design procedure accounting for the anisotropic dimensional change on sintering has been developed and successfully validated on real parts [15]. This work aims at improving the preliminary model, investigating the anisotropic dimensional changes of axial-symmetrical iron PM parts differing in geometry. The attention is focused on the effect of geometry, and in particular on the dimensional variations in the compaction plane due to the presence/size of the hole. The dimensional change of the external diameter versus the dimensional change of the internal one has been analysed in depth, aiming at defining a reliable analytical relationship dependent on geometry. Thanks to this relationship, an anisotropy parameter can be identified, depending both on geometry and on sintering conditions, which allows relating the dimensional change in the compaction plane and in the axial direction to the isotropic dimensional change. In this way the analytical model for the anisotropic behaviour can be further improved.

\section{EXPERIMENTAL PROCEDURE}

Axial-symmetric iron parts (disks and rings) have been investigated in this work, to highlight the influence of geometry. Defining the sampling two external diameters were considered (100 and $80 \mathrm{~mm}$ ) and, for rings, four different diameters of the hole; three heights $(5-10-20 \mathrm{~mm})$ for each part. Table 1 summarizes the sampling geometry.

Tab.1. Sampling geometry

\begin{tabular}{|c|c|c|}
\hline$\Phi_{\text {ext }}(\mathrm{mm})$ & $\Phi_{\text {int }}(\mathrm{mm})$ & $\mathrm{H}(\mathrm{mm})$ \\
\hline 80 & $0,20,32,50,65$ & $5,10,20$ \\
\hline 100 & $0,20,42,60,90$ & $5,10,20$ \\
\hline
\end{tabular}

All the parts were produced using a water atomized iron powder ASC100.29. 1\% Amidewax was used as compaction lubricant. Parts were cold compacted in a uniaxial press up to $7.0 \mathrm{~g} / \mathrm{cm}^{3}$ and sintered at three different temperatures, $1120^{\circ} \mathrm{C}$ in a belt furnace in a $95 \% \mathrm{~N}_{2} / 5 \% \mathrm{H}_{2}$ atmosphere, $1220{ }^{\circ} \mathrm{C}$ and $1280{ }^{\circ} \mathrm{C}$ in a vacuum furnace with nitrogen backfilling at $1000^{\circ} \mathrm{C}$, i.e. at both conventional and high sintering temperatures. Isothermal holding time was 30 minutes for all the sintering cycles.

Five specimens for each geometry and sintering condition were measured by a coordinate measuring machine (CMM). The same specimen was measured in the green and sintered state, to minimise any effect of process capability. All the surfaces were measured by continuous scan (accuracy 3.4/120 $\mu \mathrm{m} / \mathrm{sec}$ according to ISO 10360-4, 2000, which gives the scanning probe error, referred to a well-defined scan path, performed over $120 \mathrm{sec}$ [16]). The cylindrical surfaces were measured at different levels, $2 \mathrm{~mm}$ spaced. The plane surfaces were measured by circular scans, depending on the thickness of the parts: three scans for the disks, two scans for the rings with the two smallest holes, one scan for the 
rings with the two largest holes. Planes and cylindrical surfaces were calculated: processing the measured data according to a procedure described in previous works [17]. Reliable dimensions were derived from the surfaces, in terms of heights (distances between planes) and diameters of the cylindrical surfaces.

\section{RESULTS AND DISCUSSION}

\section{Anisotropic dimensional changes}

Dimensional changes in height, external diameter and internal diameter have been evaluated. The dimensional change in the direction parallel to that of the force applied during compaction is given by equation (1), being $h_{s}$ the height of the sintered part and $h_{g}$ the height of the green part

$$
\varepsilon_{h}=\frac{h_{s}-h_{g}}{h_{g}}
$$

The dimensional changes in the compaction plane are given by equations (2) and (3), being $\phi_{\text {ext } s}$ the external diameter of the sintered part, $\phi_{\text {ext } g}$ the external diameter of the green part, $\phi_{\text {int } s}$ the internal diameter of the sintered part and $\phi_{\text {int } g}$ the internal diameter of the green part.

$$
\begin{aligned}
& \varepsilon_{\phi_{\text {ext }}}=\frac{\left(\phi_{\text {ext }_{s}}-\phi_{\text {ext } g}\right)}{\phi_{\text {ext } g}} \\
& \varepsilon_{\phi_{\text {int }}}=\frac{\left(\phi_{\text {int }_{s}}-\phi_{\text {int } g}\right)}{\phi_{\text {int } g}}
\end{aligned}
$$

In the case of isotropic dimensional change, $\varepsilon_{h}=\varepsilon_{\phi \text { ext }}=\varepsilon_{\phi \text { int }}=\varepsilon_{i s o}$. The isotropic dimensional change $\varepsilon_{i s o}$ can be derived from the change in volume, as by equation (4), being $V_{s}$ the volume of the sintered part and $V_{g}$ the volume of the green part.

$$
1-\frac{V_{s}-V_{g}}{V_{g}}=\left(1+\varepsilon_{i s o}\right)^{3} \Rightarrow \varepsilon_{i s o}=\sqrt[3]{\frac{V_{s}-V_{g}}{V_{g}}+1}-1
$$

The isotropic dimensional change will be used as reference to evaluate the anisotropy for the different geometries and the different sintering temperatures.

Figures 1 and 2 show the dimensional changes measured for the different geometries at the lowest sintering temperature.

Dimensional changes shown in Figures 2 and 3 are small and highly anisotropic for all the considered parts, independently of the external diameter. Anisotropy tends to decrease on increasing the height; this effect is slightly more pronounced for parts $\phi_{\text {ext }}=80$ $\mathrm{mm}$. No definite trend due to the increase of the internal diameter is observed for parts $\phi_{\text {ext }}$ $=100 \mathrm{~mm}$, while for parts $\phi_{\text {ext }}=80 \mathrm{~mm}$ the difference between $\varepsilon_{\phi}$ ex and $\varepsilon_{\phi}$ int tends to disappear on increasing the internal diameter. 


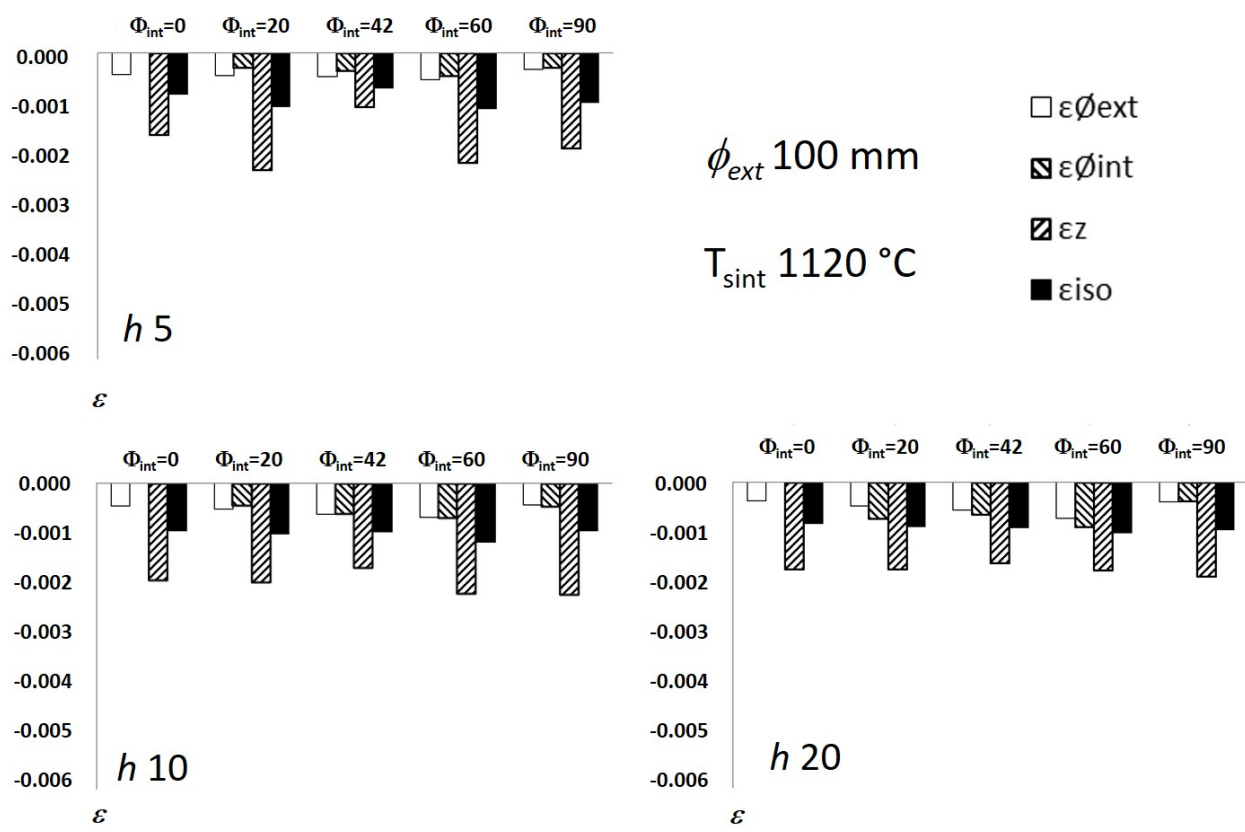

Fig.1. Dimensional changes for parts with external diameter $100 \mathrm{~mm}$ sintered at $1120^{\circ} \mathrm{C}$.

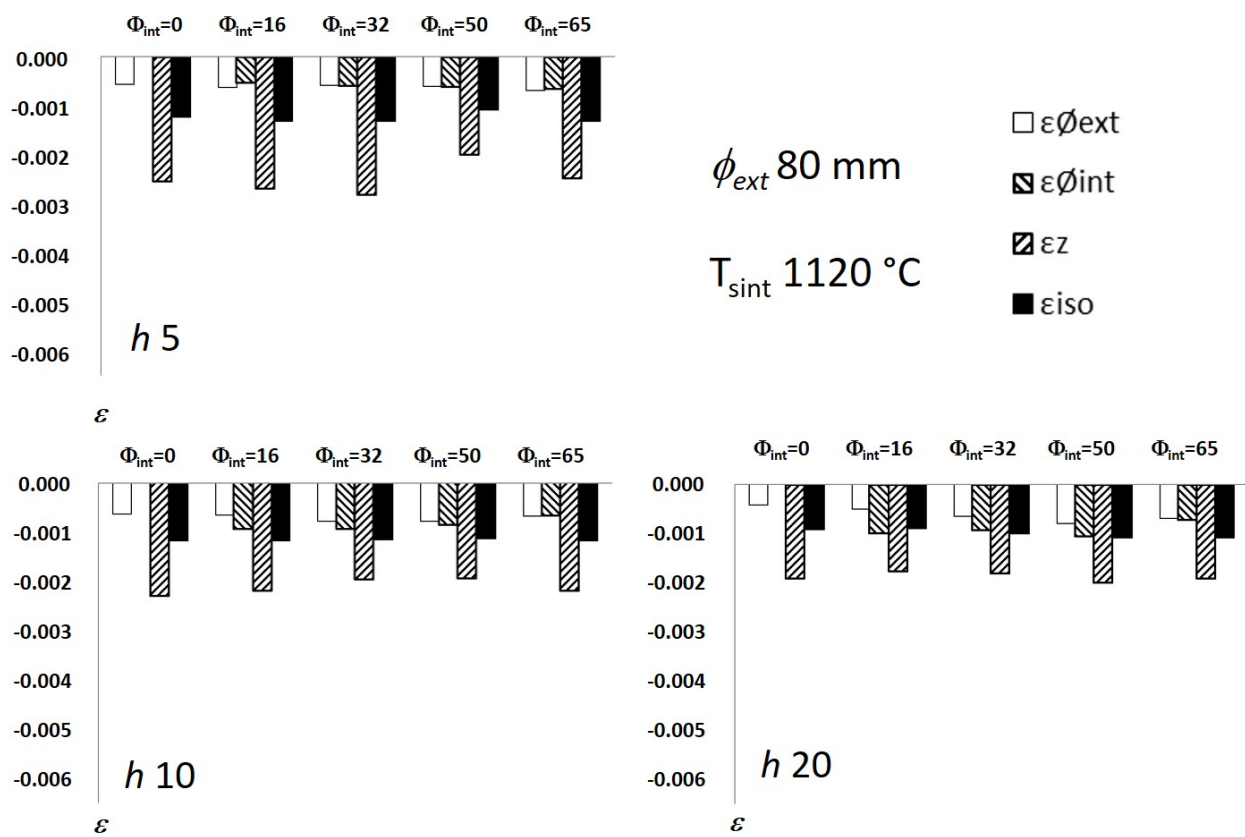

Fig.2. Dimensional changes for parts with external diameter $80 \mathrm{~mm}$ sintered at $1120^{\circ} \mathrm{C}$.

The dimensional changes measured for the different geometries at the highest sintering temperature are shown in Figures 3 and 4. 


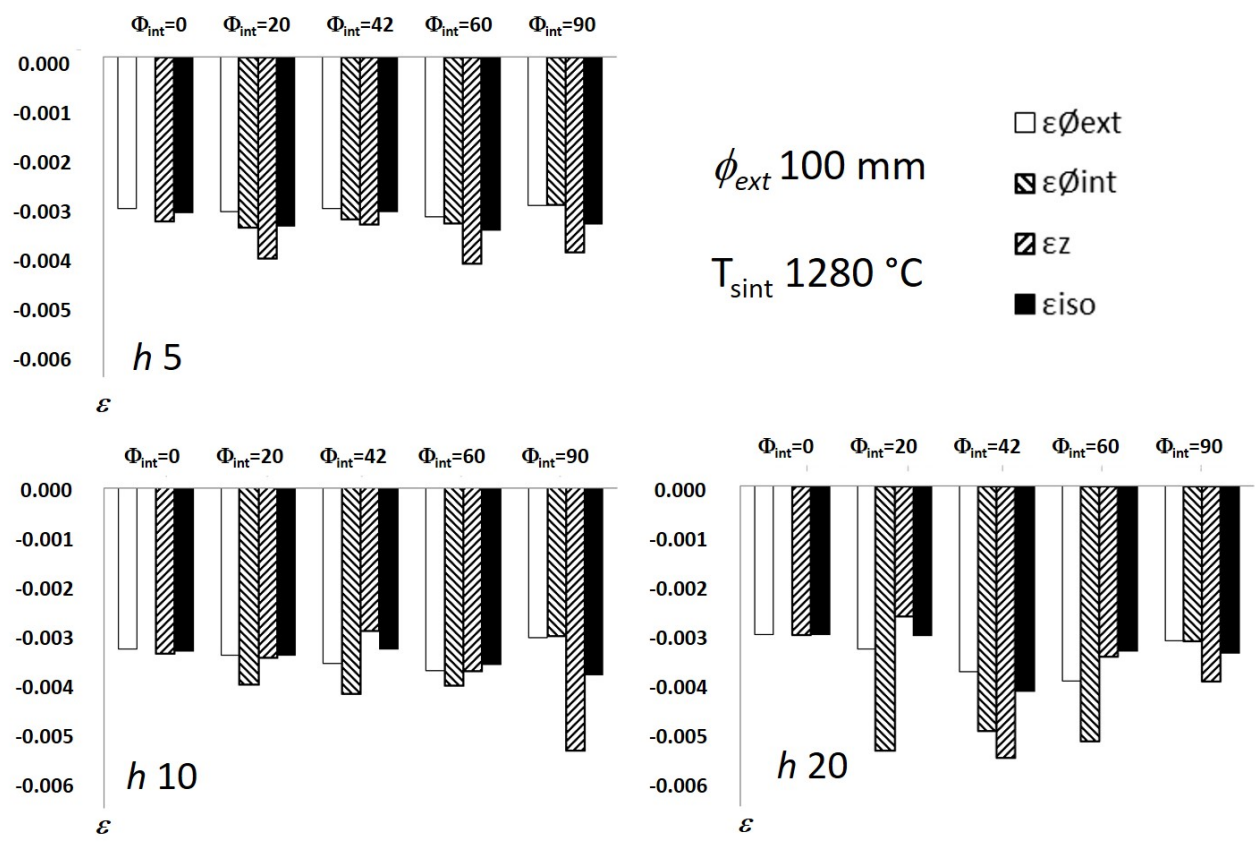

Fig.3. Dimensional changes for parts with external diameter $100 \mathrm{~mm}$ sintered at $1280^{\circ} \mathrm{C}$.

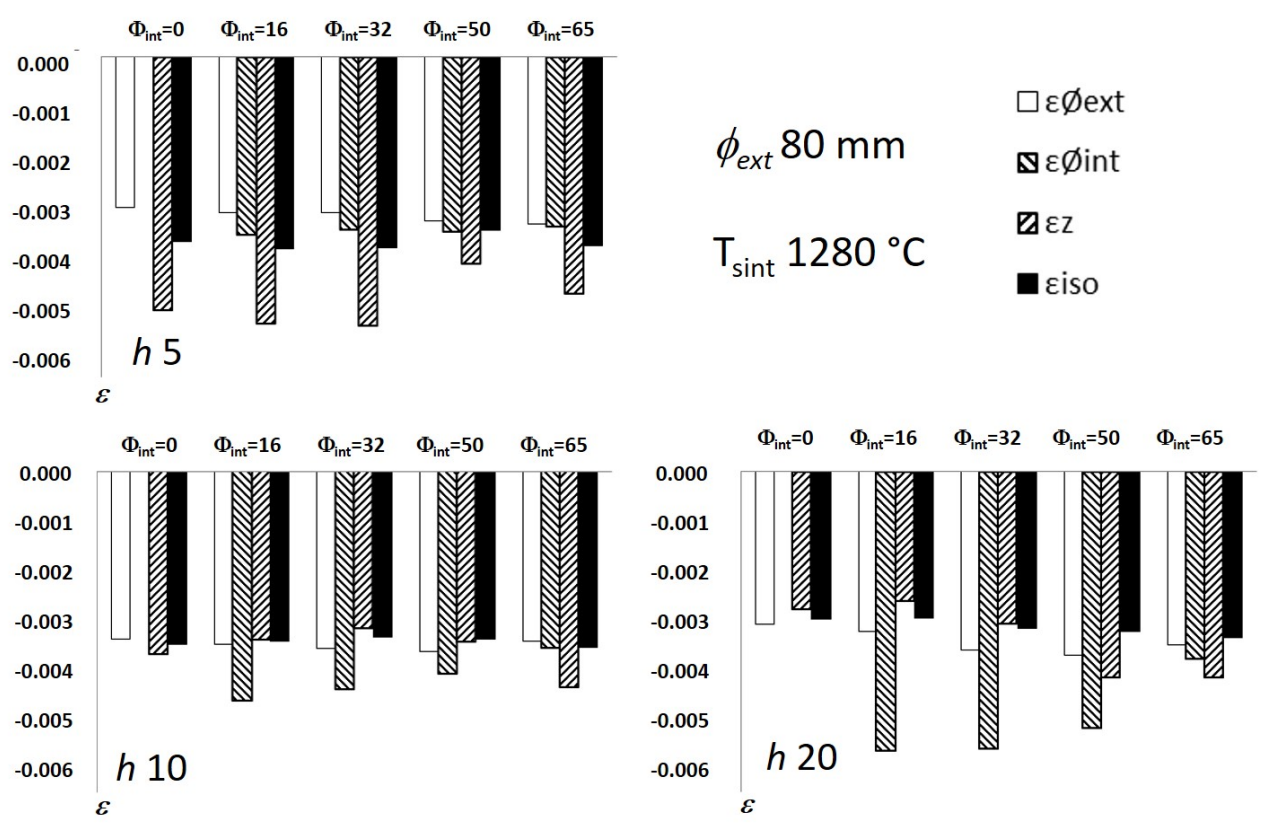

Fig.4. Dimensional changes for parts with external diameter $80 \mathrm{~mm}$ sintered at $1280^{\circ} \mathrm{C}$.

Comparing Figures 4 and 5 to Figures 2 and 3, respectively, larger dimensional changes are observed on increasing the sintering temperature, while anisotropy tends to 
decrease. A contribution to shrinkage and to its anisotropy might result from the different atmosphere that, as is well known, affects sintering behaviour of green parts significantly. To separate the effect of temperature and atmosphere, sintering tests at $1120^{\circ} \mathrm{C}$ in a vacuum furnace should be carried out. However, as shown in the following, results of sintering trials at $1220^{\circ} \mathrm{C}$ in vacuum furnace display an intermediate trend between those shown in the Figures 2 to 5 for both shrinkage and anisotropy; this result supports the conclusion that temperature has the prevailing effect.

For both values of the external diameter, the slight anisotropy tends to decrease on increasing the height for disks, while the opposite is observed for rings. Moreover, on increasing the height, larger differences between $\varepsilon_{\phi e x t}$ and $\varepsilon_{\phi i n t}$ are observed.

The dimensional change in the compaction plane is represented by the two parameters $\varepsilon_{\phi e x t}$ and $\varepsilon_{\phi i n t}$, which are differently affected by geometry and sintering conditions. The relationship between $\varepsilon_{\phi e x t}$ and $\varepsilon_{\phi i n t}$ has been investigated. Figure 5 collects all the measured data, for all the geometries and sintering temperatures.

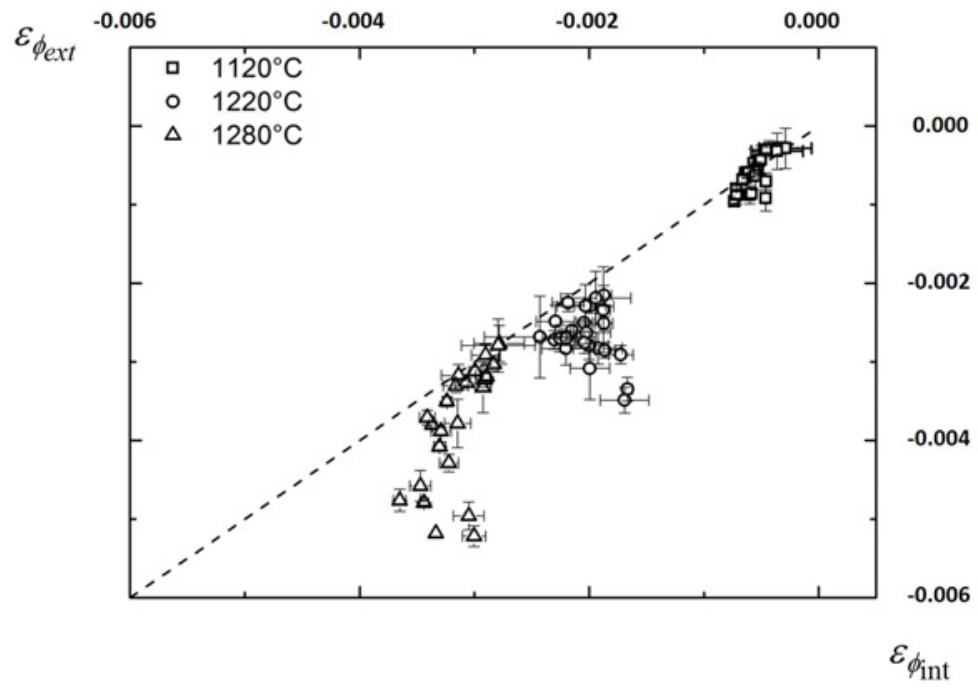

Fig.5. Dimensional change of external and internal diameter.

Figure 6 shows that the difference between the dimensional change in the internal and the external diameter increases on increasing the sintering temperature. To highlight the influence of the geometry, data have been grouped by the value of the external diameter, considering the different heights and holes size. Figures $6 \mathrm{a}$ and $6 \mathrm{c}$ show the trend for the parts with the smallest hole, external diameter $80 \mathrm{~mm}$ and $100 \mathrm{~mm}$ respectively, and Figures $6 \mathrm{~b}$ and $6 \mathrm{~d}$ show the trend for the parts with the largest hole, external diameter 80 $\mathrm{mm}$ and $100 \mathrm{~mm}$ respectively.

Figure 6 shows that linear relationships may be found for the different geometries, with different slopes, characterised by $\alpha$ coefficients according to equation (5).

$$
\varepsilon_{\phi_{\text {int }}}=\alpha \varepsilon_{\phi_{\text {ext }}}
$$


Comparing $\alpha$ coefficients, it can be observed that, for all the specimens, no significant differences can be highlighted for heights 5 and $10 \mathrm{~mm}$, while a difference is observed if height is $20 \mathrm{~mm}$. This difference tends to disappear on increasing the internal diameter (see Figure 6, $a$ vs. $b$ and $c$ vs. $d$ ). The influence of the geometry on $\alpha$ coefficient is summarised by Figure 7, where all the mean data relevant to the different geometries are collected.
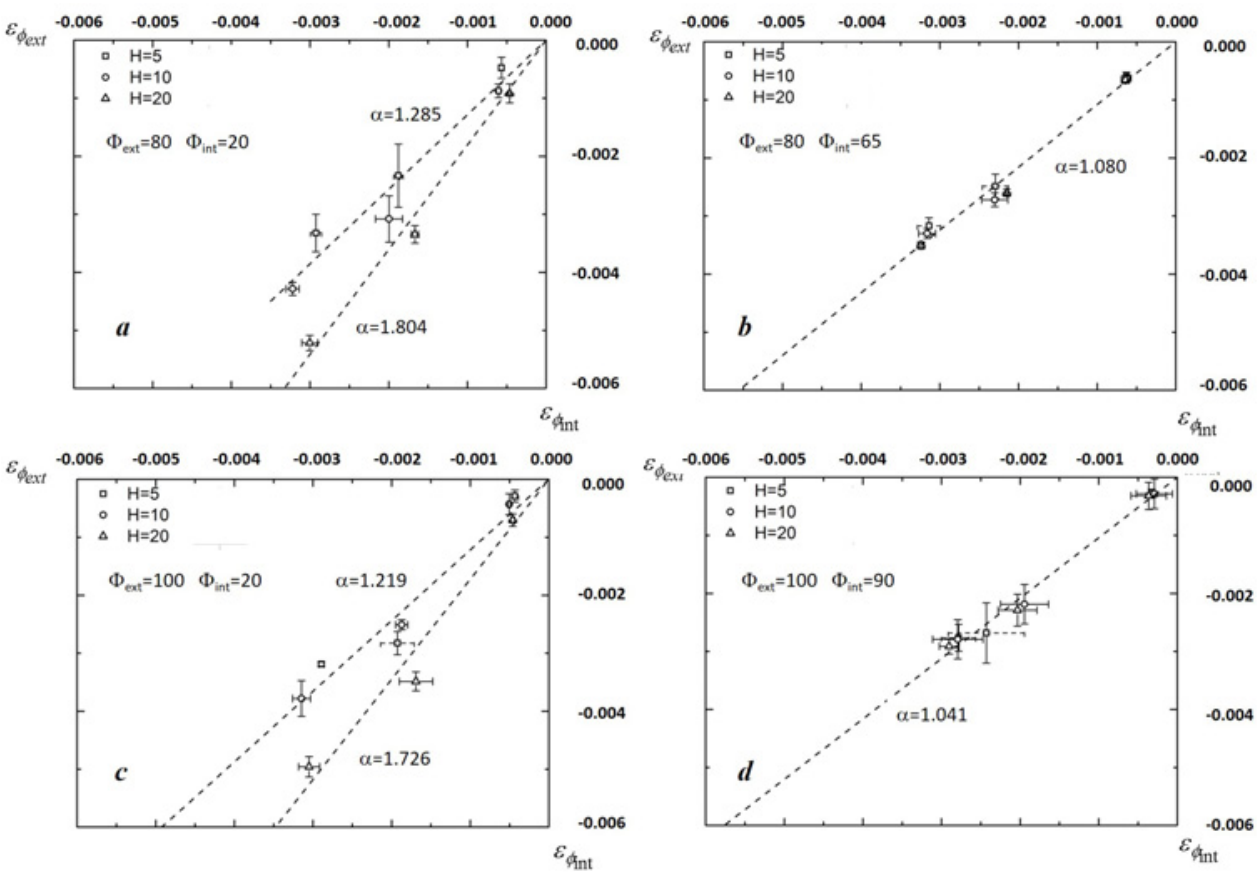

Fig.6. Relationships between $\varepsilon_{\phi e x t}$ and $\varepsilon_{\phi i n t}$ for the different geometries.

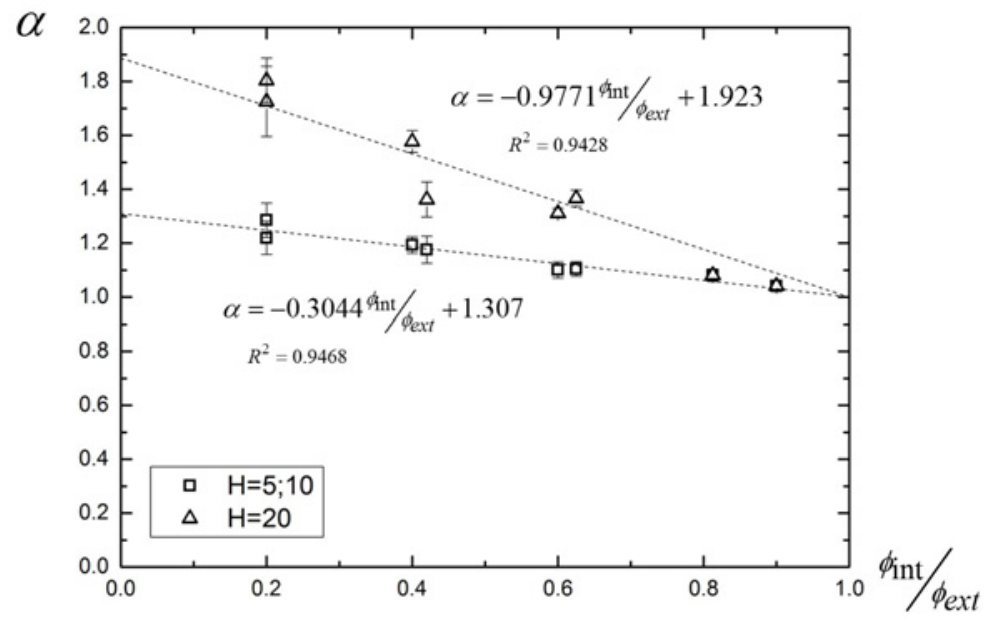

Fig.7. Influence of the geometrical parameters on $\alpha$ coefficient. 
As to Figure 7, the influence of the geometry on $\alpha$ coefficient can be synthetized by equation (6), where $m$ coefficient is a negative number depending on height

$$
\alpha=m \frac{\phi_{\text {int }}}{\phi_{\text {ext }}}+(1-m)
$$

\section{Anisotropy parameter}

Through the change in volume, the anisotropic dimensional changes can be related to the isotropic dimensional change, by means of equation (7)

$$
\begin{aligned}
& 1+\frac{V_{s}-V_{g}}{V_{g}}=\left(1+\varepsilon_{\text {iso }}\right)^{3}=\left(1+\varepsilon_{h}\right) \frac{\left(1+\varepsilon_{\phi_{\text {ext }}}\right)^{2}-R\left(1+\varepsilon_{\phi_{\text {int }}}\right)^{2}}{1-R} \\
& R=\frac{\left(\phi_{g_{\text {int }}}\right)^{2}}{\left(\phi_{g_{\text {ext }}}\right)^{2}}
\end{aligned}
$$

The anisotropy of dimensional changes is given by the difference between the dimensional changes in the direction parallel to the compaction force (height) and the dimensional changes in the compaction plane (diameters). Using equations (7) and (5), the two contributions may be distinguished, according to equation (8):

$$
\frac{\left(1+\varepsilon_{i s o}\right)^{3}}{\left(1+\varepsilon_{h}\right)}=\frac{\left(1+\varepsilon_{\phi_{e x t}}\right)^{2}-R\left(1+\alpha \varepsilon_{\phi_{e x t}}\right)^{2}}{1-R}
$$

According to Figure 8, the "distance" between the isotropic and the anisotropic behaviour can be used to estimate the entity of the anisotropic dimensional changes.

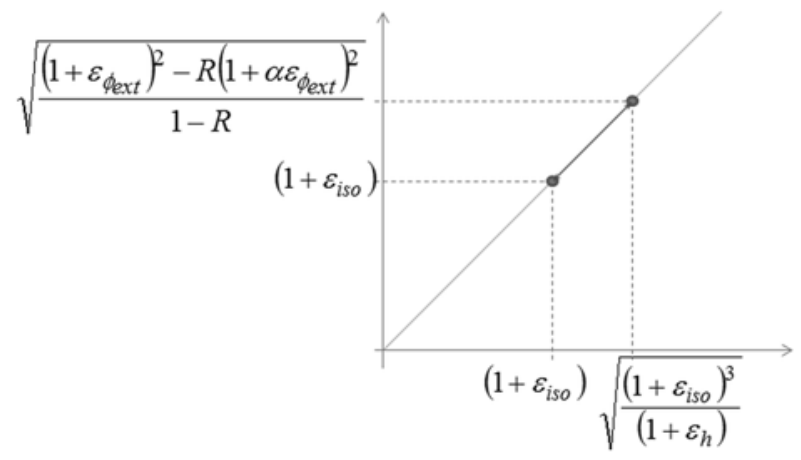

Fig.8. Representation of the anisotropic behaviour as the distance from the isotropic one.

On the basis of the previous consideration, using the distance above, an anisotropy parameter $\mathrm{K}$ is proposed to estimate the entity of anisotropy of dimensional changes, given by equation (9) 


$$
K=\frac{\sqrt{\frac{\left(1+\varepsilon_{\phi_{e x t}}\right)^{2}-R\left(1+\alpha \varepsilon_{\phi_{e x t}}\right)^{2}}{1-R}}-\left(1+\varepsilon_{\text {iso }}\right)}{\varepsilon_{\text {iso }}}
$$

eqn. (1)

Expanding the square terms and introducing coefficients $\gamma$ and $\delta, K$ is given by

$$
\begin{aligned}
& \gamma=\frac{\left(1-\alpha^{2} R\right)}{(1-R)} \\
& \delta=2 \frac{(1-\alpha R)}{(1-R)} \\
& K=\frac{\sqrt{\gamma\left(\varepsilon_{\phi_{\text {ext }}}\right)^{2}+\delta \varepsilon_{\phi_{\text {ext }}}+1}-\left(1+\varepsilon_{\text {iso }}\right)}{\varepsilon_{\text {iso }}}
\end{aligned}
$$

For the different geometries $K$ is plotted vs. $\varepsilon_{i s o}$ in Figure 9. The most significant geometries are shown, among all those evaluated; intermediate trends are shown in the missing ones.
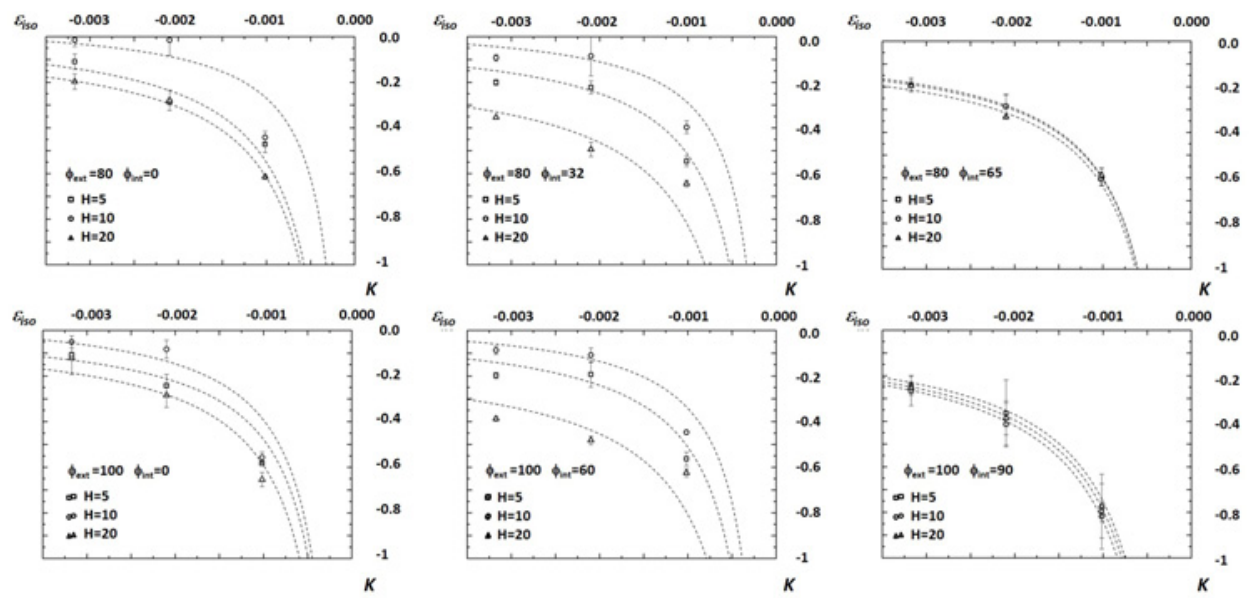

Fig.9. $K$ vs. $\varepsilon_{i s o}$ for the different geometries.

The influence of height, as shown in Figure 9, is significant for disks, even more significant for rings with a quite large hole and almost insignificant for the rings with the largest hole. This trend is particularly evident for the specimens characterized by $\phi_{\text {ext }}=80$ $\mathrm{mm}$. The highest anisotropy is generally found for the highest specimens.

In previous works [14, 15], a relationship between $K$ and $\varepsilon_{i s o}$ was found experimentally, considering rings differing for dimensions, material and sintering temperatures, given by equation (11) 


$$
K=\frac{\varepsilon_{i s o}}{\left|\varepsilon_{i s o}\right|} \exp \left(\frac{A}{\left|\varepsilon_{i s o}\right|^{n}}+B\right)-1
$$

where $\varepsilon_{i s o}$ is the parameter mainly dependent on the material and process conditions, while $A, B$ depend on geometry, and $\mathrm{n}$ is equal to $2 / 3$, being the best fitting value. The ratio between $\varepsilon_{i s o}$ and its absolute value accounts for shrinking or swelling, being simply equal to -1 for shrinking systems and +1 for swelling systems.

This relationship was found considering a large amount of data, in an interval comprehending extremely small to very high isotropic dimensional changes. In the present work, due to the lower amount of data, equation (11) does not fit so accurately the experimental values, and the reliability of values found for the constants $A$ and $B$ is low. It has to be considered, however, that fitting by equation (11) was mainly driven by the presence of data relevant to extremely small dimensional changes, corresponding to experimental process conditions, which are not industrial $\left(\mathrm{T}_{\text {sint }} 1000^{\circ} \mathrm{C}\right)$. Concerning the data in the present work, corresponding to industrial process conditions, the fitting curve may be described as a first approximation to an easier relationship between $K$ and $\varepsilon_{i s o}$, expressed by equation (12)

$$
K=\frac{C}{\varepsilon_{i s o}}+D
$$

where $C$ and $D$ depend on the geometric parameters, as shown in Figure 10.

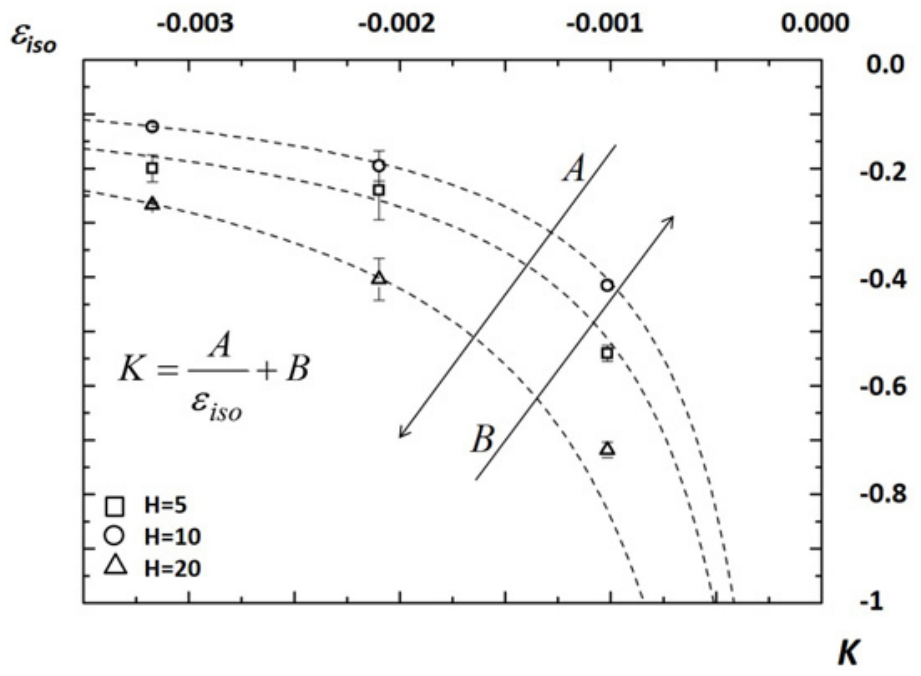

Fig.10. The relationship between $K$ and $\varepsilon_{i s o}$.

Considering the graphs of all the geometries, $C$ and $D$ belong to the following intervals: $C \varepsilon\left[3.5 \cdot 10^{-4}, 8.5 \cdot 10^{-4}\right], D \varepsilon[-0.1,0.1]$. 
This kind of representation highlights that further investigation is needed to improve the knowledge on the influence of geometry on anisotropic dimensional change. As previously observed, the variation of the anisotropy parameter versus the change in height is not monotonic, and it is not monotonic versus the change in the dimensions in the compaction plane, as shown in Figure 11, where $K$ is reported versus the ratio between the internal and external diameter.

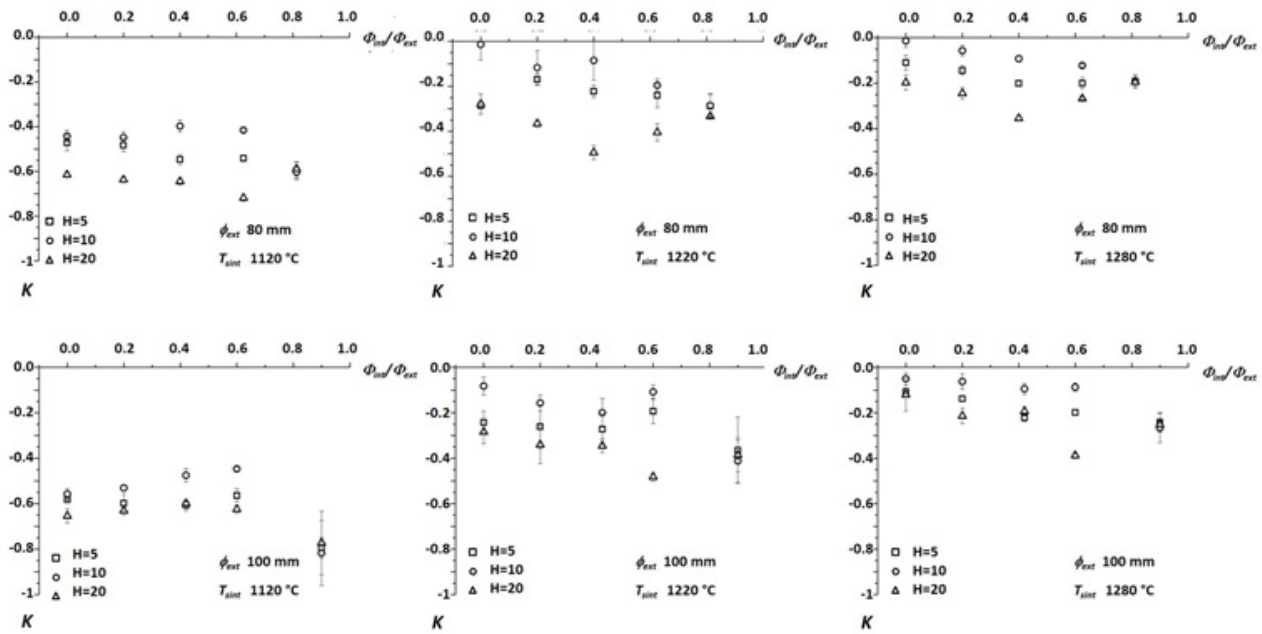

Figure 11. $K$ versus $\Phi_{\text {int }} / \Phi_{\text {ext }}$ for the different sintering temperatures.

Aiming at understanding this trend, future work will further improve the definition of the anisotropy parameter, evaluating the variables mainly responsible of the anisotropic dimensional change during the whole press and sinter process. The distribution of axial and radial pressure during compaction will be considered, as well as the heating rate during sintering, in addition to the sintering temperature.

\section{CONCLUSION}

This work investigated the anisotropic dimensional change on sintering aiming at proposing an analytical model based on experimental data. Axial-symmetric iron parts differing in geometry and sintering conditions have been considered. Main results are the following.

- Dimensional changes increase on increasing the sintering temperature, while anisotropy tends to decrease.

- Even if tests at $1120^{\circ} \mathrm{C}$ were carried out in a belt furnace in endogas atmosphere, while those at higher temperatures were carried out in a vacuum furnace, all data fits an inverse correlation shrinkage and its anisotropy. This suggests that sintering temperature has the prevailing effect.

- Concerning the dimensional changes in the compaction plane, the difference between the dimensional change in the internal and the external diameter increases on increasing the sintering temperature.

- A linear relationship between the dimensional change in the internal and the external diameter has been found, 


$$
\varepsilon_{\phi_{\text {int }}}=\alpha \varepsilon_{\phi_{\text {ext }}}
$$

where $\alpha$ coefficient depends on the geometry according to the relationship

$$
\alpha=m \frac{\phi_{\text {int }}}{\phi_{\text {ext }}}+(1-m)
$$

$m$ coefficient being a negative number depending on height.

- An anisotropy parameter $K$ has been proposed, which allows relating the dimensional change in the compaction plane and in the axial direction to the isotropic dimensional change. An analytical model has been proposed fitting the experimental data

$$
K=\frac{C}{\varepsilon_{i s o}}+D
$$

where $C$ and $D$ depend on the geometric parameters. The variation of the anisotropy parameter versus the change in height is not monotonic, and it is not monotonic versus the change in the dimensions in the compaction plane.

- Future work will further improve the definition of the anisotropy parameter by means of the variables mainly responsible of the anisotropic dimensional change during the whole press and sinter process.

\section{References}

[1] Zavaliangos, A., Bouvard, D.: The international Journal of Powder Metallurgy, vol. 36, 2000, no. 7, p. 58

[2] Wanibe, Y., Yokoyama, H., Itoh, T.: Powder Metallurgy, vol. 33, 1990, no. 1, p. 65

[3] Griffo, A., Ko, J., German, RM. In: Advances in Powder Metallurgy and Particulate Materials. Vol. 3. Princeton (NJ) : Metal Powders Industry Federation, 1994, p. 221

[4] Raman, R., Zahrah, TF., Weaver, TJ., German, RM. In: Advances in Powder Metallurgy and Particulate Materials 1999. Vol. 1. Princeton (NJ) : Metal Powder Industries Federation, 1999, p. 115

[5] Cristofolini, I., Menapace, C., Cazzolli, M., Rao, A., Pahl, W., Molinari, A.: Journal of Materials Processing Technology, vol. 212, 2012, p. 1513

[6] Cristofolini, I., Molinari, A., Pilla, M., Menapace, C., Larsson, M.: The International Journal of Powder Metallurgy, vol. 48, 2012, no. 4, p. 33

[7] Olevsky, EA., German, RM.: Acta Materialia, vol. 48, 2000, p. 1153

[8] Olevsky, EA., German, RM.: Acta Materialia, vol. 48, 2000, p. 1167

[9] Vo, A., Guillot, M. In: Advances in Powder Metallurgy and Particulate Materials 1999. Vol. 1. Princeton (NJ) : Metal Powder Industries Federation, 1999, p. 123

[10] Menapace, C., Larsson, M., Torresani, E., Cristofolini, I., Molinari, A. In: Proceedings of PM2012 Powder Metallurgy World Congress and Exhibition. Yokohama (Japan), 14-18 October 2012, 16D-T3-17

[11] Cristofolini, I., Selber, F., Menapace, C., Pilla, M., Molinari, A., Libardi, S. In: Proceedings of EuroPM 2012 Congress \& Exhibition. Vol. 1. Basel (CH), September 16-19, 2012, p. 23

[12] Molinari, A., Menapace, C., Torresani, E., Cristofolini, I., Larsson, M.: Powder Metallurgy, vol. 56, 2013, no. 3, p. 189

[13] Cristofolini, I., Corsentino, N., Pilla, M., Molinari, A., Larsson, M. In: Advances in 
Powder Metallurgy and Particulate Materials. Vol. 11. Princeton (NJ) : Metal Powders Industry Federation, 2013, p. 49

[14] Cristofolini, I., Corsentino, N., Molinari, A., Larsson, M.: International Journal of Precision Engineering and Manufacturing, vol. 15, 2015, no. 9, p. 1865

[15] Cristofolini, I., Corsentino, N., Molinari, A., Larsson, M. In: Advances in Powder Metallurgy and Particulate Materials. Vol. 1. Princeton (NJ) : Metal Powders Industry Federation, 2014, p. 115

[16] ISO 10360-4, 2000. Geometrical Product Specifications (GPS) - Acceptance and reverification tests for coordinate measuring machines (CMM) - Part 4: CMMs used in scanning measuring mode

[17] Cristofolini, I., Rao, A., Menapace, C., Molinari, A.: Journal of Materials Processing Technology, vol. 210, 2010, p. 1716 